\title{
Subspecific Divergence in the Black Lemur's Low-Pitched Vocalizations
}

\author{
M. Gamba ${ }^{*}$ and C. Giacoma
}

Department of Animal and Human Biology, University of Torino, Italy

\begin{abstract}
Previous studies offered very preliminary information on the vocal repertoire of Eulemur macaco macaco and Eulemur macaco flavifrons. They agreed on the fact that both subspecies emit low-pitched vocalizations, called grunts, of different duration. Through all-occurrence and focal animal observations, we recorded the vocal activity of 31 black lemurs (12 E. m. macaco and 19 E. m. flavifrons) housed in 7 institutions, both in Europe and in Madagascar. We measured both temporal and spectral properties to describe long grunts quantitatively. We extracted acoustic parameters in the perspective of the source-filter theory of sound production. From spectrograms, we measured call duration and 6 larynxrelated features and, using Linear Predictive Coding spectra, we measured four vocal tract related acoustic properties. Our quantitative analysis has statistical support for the classification of long grunts. Using individual mean values and multivariate Discriminant Function Analysis we have been able to successfully classify $96.8 \%$ of the vocal signals to the subspecies of the emitter. Acoustic cues of both larynx-related and vocal tract-related acoustic parameters offered support for sub-specific recognition potential. However, univariate analyses showed that formants should be providing listeners with subspecies-specific information.
\end{abstract}

\section{INTRODUCTION}

The identification of animal species using acoustic characters of vocalization represents a valuable non-invasive method for the study and management of wild populations, especially in those cases in which intergradations of character may occur because of hybridization or because of geographic variation in morphological traits.

The black lemur (Eulemur macaco) is a medium-sized prosimian species whose distribution is limited to the northwestern areas of Madagascar, including the two adjacent islands of Nosy Komba and Nosy Be. It comprises two subspecies, the black lemur (Eulemur macaco macaco) and the blueeyed black lemur (Eulemur macaco flavifrons). Both subspecies are sexually dichromatic. The northern limit of the black lemur's distribution is the Mahavavy river. Other limits are thought to be the Tsaratanana Massif (East) and the Andranomalaza river (South), as shown in Fig. (1) [1]. Males are typically black or dark brown with prominent ear tufts of long black hair. Females show a brownish dorsal coat and white or creamy ventral coat. Ear tufts are usually creamy or white. Both sexes have a black face with dark grey areas. This subspecies is classified as Endangered (EN A2cd) [1]. The blueeyed black lemur or Sclater's black lemur (E. m. flavifrons) occurs in a small area of sub-humid forest in northwest Madagascar, to the northern boundary of the Andranomalaza river and up to the Maevarano river (South) and the Sandrakota river (East), as shown in Fig. (1) $[1,2]$. The taxonomic validity of this subspecies was recently confirmed [3-5] and it was only rediscovered in recent years [6-8].

In the border region of the distribution area of the two subspecies intermediate forms have been described $[2,8,9]$.

*Address correspondence to this author at the Department of Animal and Human Biology, University of Torino, Italy;

E-mail: marco.gamba@unito.it
The existence of an intergradation zone and a geographical cline in subspecific traits between the two forms over the Manongarivo Mountain has been suggested [8]. More information is needed to clarify other possible hybridization zones occurring over the distribution of both subspecies [2].

Few data are available about differences in body size between the two subspecies. Black lemurs (E. m. macaco) are supposed to have a head-body length of $39-45 \mathrm{~cm}$ and a body weight 2.0-2.9 Kg [1]. Blue-eyed black lemurs have a head-body length of $39-45 \mathrm{~cm}$ and body weight $1.8-1.9 \mathrm{Kg}$ [1].

Previous studies on the behaviour and vocal communication of this species have provided little quantitative data. Macedonia and Stanger (1994) [10] reported 8 vocal types in the repertoire of the black lemur colony maintained at Duke University Primate Center (DUPC). Most of their analysis agrees with the descriptions given by Petter and CharlesDominique [11] who collected data from wild specimens. Studies conducted on the captive colony at the Louis Pasteur University Primatology Center (Strasbourg, France) reported 18 different acoustic signals produced by the study animals [12].

In all previous studies the presence of a low-pitched vocalizations, named long grunt (long duration grunt or simple long grunt), is reported.

Quantitative investigations on the acoustic structures of low-pitched vocalizations in lemurs provided evidence that utterances emitted by different Eulemur species possess distinctive characteristics $[13,14]$.

All true lemurs (Eulemur sp.) produce vocalizations showing very low fundamental frequencies. They are generally called grunts and also occur in association with other acoustic units $[10,12,13]$. 
Among those grunts that occur simply as a train of pulses at low frequency, it has been suggested that one distinct vocal type can be recognised by its duration, the long grunt [15]. This vocalization is suitable for measuring both larynxrelated and vocal tract-related parameters and for this reason it was chosen for the present study.

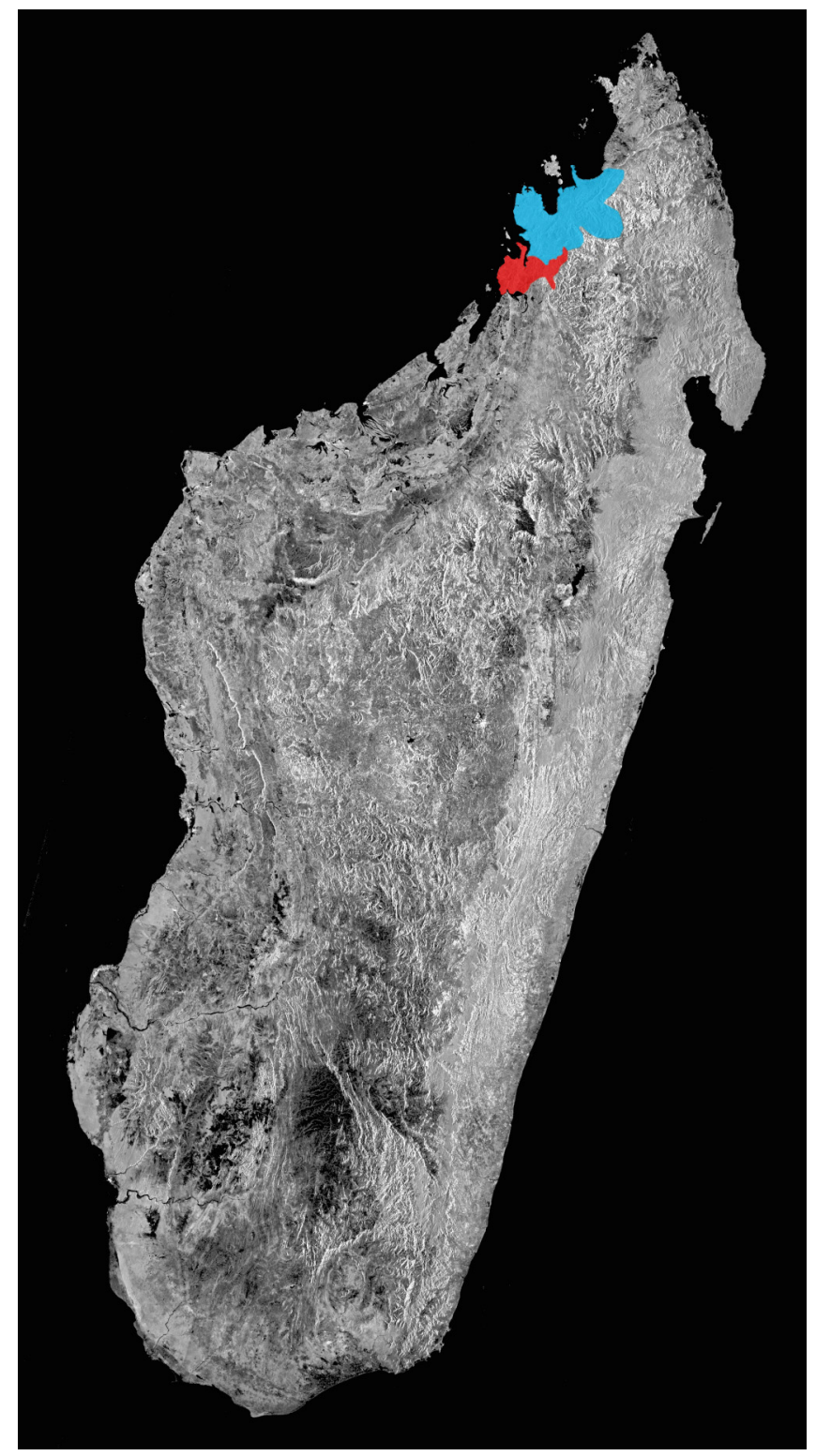

Fig. (1). Distribution of the black lemur (E. m. macaco in blue) and the blue-eyed black lemur (E. m. flavifrons in red).

In this paper we will investigate the presence of subspecific potential in the acoustic structure of long grunts emitted by black lemurs and blue-eyed black lemurs. We will also use the vocal tract dependent acoustic cues to estimate the length of the supralaryngeal vocal tract of the study individuals.

\section{MATERIALS AND METHODOLOGY}

\section{Subjects}

All vocalizations were recorded from black lemurs in captivity. The following institutions were visited, between July 1999 and May 2005, for the present study: Parco Natura
Viva - Garda Zoological Park (Italy), Mulhouse Zoo (France), Parc Botanique et Zoologique de Tsimbazaza (Madagascar), Banham Zoo (U.K.), Linton Zoo (U.K.), Apeldoorn Apenheul (The Netherlands), St. Louis Zoo (USA). All 7 study groups received daily diets mainly consisting of fruit and vegetables, and water ad libitum. All subjects were maintained on a natural light/dark daily cycle. All subjects were treated in accordance with animal care guidelines and international regulations.

Recordings of twelve black lemurs (E. m. macaco) and 19 blue-eyed black lemurs (E. m. flavifrons) were used in the analysis.

\section{Vocalization Recording}

Calls were recorded with Sony TCD-D100 and TCD-D8 digital tape recorders, equipped with Sony EMS-907 microphones. When necessary, we limited environmental noise interference by using a shotgun microphone, Sennheiser ME88. The sample rate should be slightly higher than twice the maximum frequency of the vocalizations that will be analyzed. Therefore, for the maximum signal frequency of $8000 \mathrm{~Hz}$ that we analysed, a sample rate of $44100 \mathrm{~Hz}$ was considered appropriate [16].

We considered only groups where we had recorded for, at least, 2 sessions of 1 hour each. To increase the number of recorded vocalizations, we used both all-occurrence and focal animal sampling methods [17]. Depending on the housing conditions, vocalizations were recorded in outdoor and indoor enclosures. We recorded all of the spontaneously occurring vocalizations, without the use of visual or acoustic playbacks.

\section{Acoustic Analysis}

Recordings were digitalized at a sample rate of $44100 \mathrm{~Hz}$ (16-bit resolution, mono format) with SoundForge 7 software (Madison Media Software Inc., Sony Corporation of America, USA). After a preliminary qualitative analysis of the entire recordings, we selected and saved into separate files all vocalizations whose recording quality allowed their subsequent quantitative descriptions and where we knew emitter identity.

The total sample of selected vocalizations comprised 441 black lemurs' (E. m. macaco) long grunts emitted by 6 females and 6 males, belonging to 3 groups.

For the blue-eyed black lemurs' (E. m. flavifrons) the sample comprised 223 long grunts emitted by 8 females and 11 males (belonging to 7 groups).

The analytical approach was based on a source-filter theory perspective because both vocal fold vibration and supralaryngeal filtering contribute to shaping vocalizations in primates [18-21], including lemurs [22-24].

Therefore we measured duration of the whole vocal emission (DUR); three larynx-related features (Fig. 2) - average fundamental frequency $(\mathrm{MeF} 0)$, minimum fundamental frequency (MiF0), maximum fundamental frequency (MaF0, Fig. 2B); three vocal tract-related acoustic features average first formant (F1), average second formant (F2), 
average third formant (F3, Fig. 2A). Mean values were calculated averaging all points detected in the fundamental frequency contour and in each formant contour.

We measured acoustic properties of the vocalizations using Praat $[21,25,26]$. Additional spectral analyses were performed with Canary [27].

To detect source features (MeF0, MiF0, MaF0), Fast Fourier transforms were generated for all calls (frequency range: $0-12000 \mathrm{~Hz}$; maximum: $50 \mathrm{~dB} / \mathrm{Hz}$; dynamic range: 30 $\mathrm{dB}$; pre-emphasis: $6.0 \mathrm{~dB} / \mathrm{Oct}$; dynamic compression: 0.0 ). The actual variation of the fundamental frequency was measured using the autocorrelation method ["Sound: To pitch (ac)..."] after adjusting the analysis parameters according to the range of variation in each of the vocalization [13].

Formants (F1, F2, F3) were studied using linear predictive coding (LPC, Formant settings: maximum formant 9500 $\mathrm{Hz}$, number of formants 7.0 , window length 0.06 , dynamic range $45.0 \mathrm{~dB}$ ). We usually selected 3 formants (out of the 67 formants highlighted on the screen). Two methods were used in order to verify formant pattern predicted by LPC. First, formant analyses were superimposed over the signal spectrogram. Second, autocorrelation-based LPC spectra were overlaid on independently derived FFT spectra of the same frames to verify the goodness of the LPC analysis. The formant pattern fitting was inferred during a step-by-step monitored process, where the operator could interrupt the analysis and modify the analysis parameters (maximum formant and number of formants) to obtain the optimal fitting. After formant measurements, formant dispersion (DF) was calculated using the formula proposed by Fitch [20]. A custom-modified Praat script was used to automate file opening and editing and file saving of the measurements [28].

\section{Statistical Analysis of Acoustic Parameters}

To avoid pseudoreplication, statistical analyses were carried out on the individual mean values of each acoustic property. This also minimized minor changes, potentially occurring because of momentary circumstances, in the spectral or temporal characteristics.

Analysis of variance (ANOVA) was used to test differences between subspecies in single acoustic parameters. We then used Discriminant Function Analysis (DFA) to identify linear combinations of predictor variables that maximize the differences among vocal types. DFA has been successfully used in previous studies of non-human primate vocal repertoires $[13,29]$. DFA was run using a stepwise procedure and F-value thresholds for acceptance or rejection of independent variables were set at $\mathrm{F}=3.84$ and $\mathrm{F}=2.71$ in all analyses.

After that, we estimated vocal tract lengths of the emitters using resonance frequencies measured from the long grunts [30]. F1, F2, F3 frequency values were plotted against $\left(2 i^{-1}\right) / 2$ increments of the formant spacing, where $i$ corresponds to the formant number. A linear regression line was fitted to the values, using an intercept equal to 0 . This method returns estimates of minimum $\Delta \mathrm{F}(\min \Delta \mathrm{F})$, thus estimated maximum vocal tract length (maxVTL) was calculated using $\max V T L=c / 2(\min \Delta \mathrm{F})[30]$, where $c$ is the speed of sound in air (approximated as $350 \mathrm{~m} / \mathrm{s}$ in a mammal's vocal tract [19]).

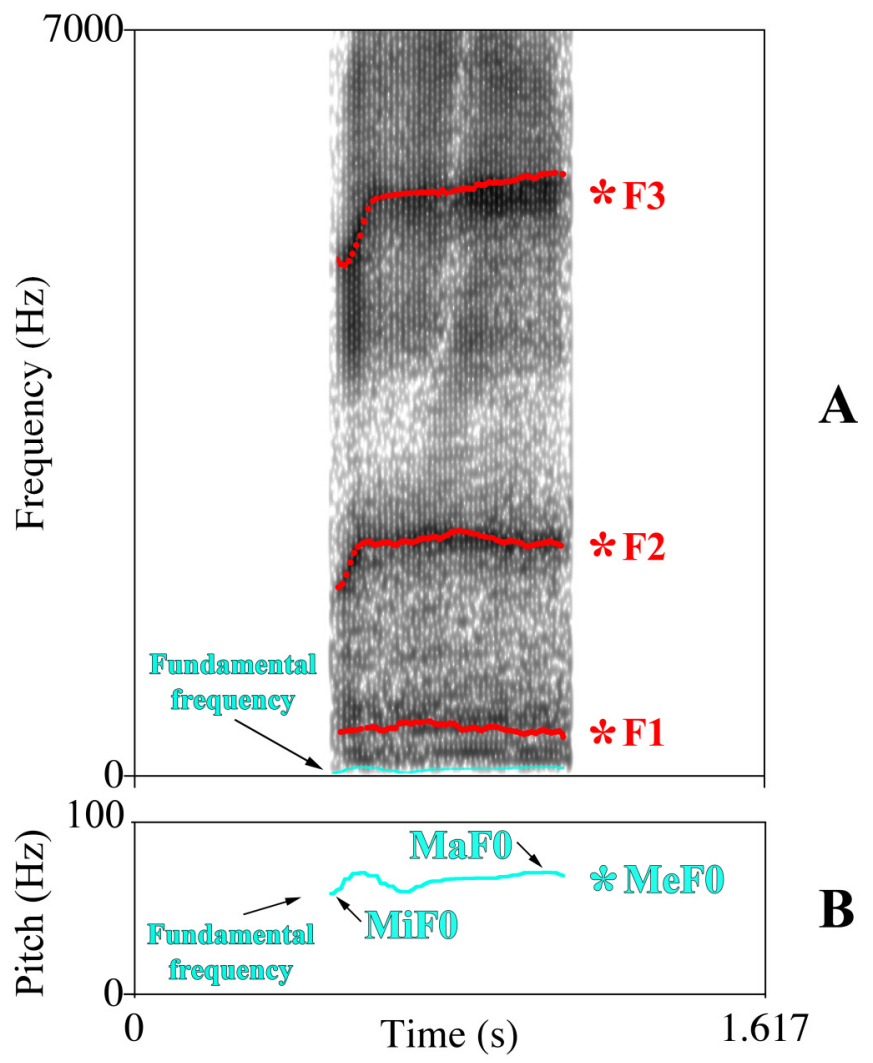

Fig. (2). Spectrogram of a long grunt with formants and pitch (Fundamental frequency) contours overlaid (A) and pitch contour showing Fundamental frequency variation (B). This figure was obtained by drawing and exporting from Praat into a graphic program: spectrogram, pitch-extraction and formant-extraction contours of a long grunt. In 2A, Asterisks indicate average values of F1, F2 and F3. In $2 \mathrm{~B}$, arrows indicate minimum $(\mathrm{MiF} 0)$ and maximum (MaF0) Fundamental frequencies, the asterisk indicates calculated average Fundamental frequency (MeF0).

\section{RESULTS}

\section{Comparison of Long Grunts Between Subspecies}

We initially tested homogeneity of variance between the datasets of the two subspecies $(0.077<\mathrm{p}<0.756)$, using ANOVA.

Table 1. Mean and Standard Deviation of the Acoustic Parameters Per Subspecies

\begin{tabular}{|c|c|c|c|}
\hline & DUR (s) & MeF0 (Hz) & F1 (Hz) \\
\hline \hline E. m. macaco & $0,367 \pm 0,082$ & $75 \pm 8$ & $1035 \pm 247$ \\
\hline E. m. flavifrons & $0,512 \pm 0,251$ & $73 \pm 8$ & $544 \pm 225$ \\
\hline & $\mathbf{F 2}(\mathbf{H z})$ & $\mathbf{F 3} \mathbf{( H z )}$ & $\mathbf{D F} \mathbf{( H z )}$ \\
\hline \hline E. m. macaco & $3108 \pm 351$ & $5165 \pm 303$ & $2000 \pm 73$ \\
\hline E. m. flavifrons & $2401 \pm 346$ & $4792_{ \pm} 243$ & $2095 \pm 107$ \\
\hline
\end{tabular}

Number of average individual values considered is 12 for E. m. macaco and 19 for $E$. m. flavifrons. Parameters are: duration (DUR), average fundamental frequency (MeF0), average first formant (F1), second formant (F3), third formant (F3), formant dispersion (DF). 
ANOVA yielded significant results for $\mathrm{F} 1 \quad(\mathrm{~N}=31$, $\mathrm{F}=32.438, \mathrm{p}<0.001), \mathrm{F} 2 \quad(\mathrm{~N}=31, \mathrm{~F}=30.283, \mathrm{p}<0.001, \mathrm{~F} 3$ $(\mathrm{N}=31, \mathrm{~F}=14.302, \mathrm{p}=0.001)$ and $\mathrm{DF}(\mathrm{N}=31, \mathrm{~F}=7.230$, $\mathrm{p}=0.012$ ).

We applied Discriminant Function Analysis to verify the hypothesis that the two E. macaco subspecies produce long grunts identifiable by quantitatively measuring the temporal, spectral and formant parameters. Therefore we determined which variables contributed to the discrimination of subspecific utterances.

Results showed statistically significant differences among long grunts $\left(\mathrm{N}=31\right.$, Wilk's Lambda $=0.244, \mathrm{~F}_{4,26}=$ $20.125, p<0.001)$ and most average values for the different call types for each lemur were correctly grouped to the proper category. The F levels identified by DFA showed that four acoustic parameters $(\mathrm{F} 2, \mathrm{~F}=50.772$, Tol. $=0.654$; DF, $\mathrm{F}=6.375$, Tol. $=0.858 ; \mathrm{MeF} 0, \mathrm{~F}=11.056$, Tol. $=0.670$, DUR, $F=5.132$, Tolerance $=0.792$ ) contributed to the discrimination of each vocal category. One Discriminant function was identified by the analysis and showed statistical differences among subspecies (ANOVA; $\mathrm{N}=31, \mathrm{~F}=89.788$, $\mathrm{p}<0.001$ ). Classical stepwise Discriminant Function Analysis and cross-validated Discriminant function analysis correctly classified respectively $96,8 \%$ and $93,5 \%$ by vocal type. All black lemurs (E. m. macaco) were correctly classified into the appropriate category in the classical stepwise analysis. All blue-eyed black lemurs were correctly classified except for one individual, who was incorrectly classified into the other subspecies. In the cross-validated procedure, one further E. m. macaco individual was incorrectly classified into the other subspecies. In cross-validation, each case is classified by the functions derived from all cases other than the case considered.

\section{Vocal Tract Length Estimates}

Applying the Reby and McComb method, estimated minimum formant spacing was $2034 \pm 119 \mathrm{~Hz}$ for black lemurs (E. m. macaco) and $1893 \pm 101 \mathrm{~Hz}$ for blue-eyed black lemurs (E. m. flavifrons). These minimum distances between formants correspond to nasal tract lengths of $8.6 \pm 0.5 \mathrm{~cm}$ and $9.3 \pm 0.5 \mathrm{~cm}$, in E. m. macaco and E. m. flavifrons respectively.

Significant differences were found when we compared the nasal tract length estimates between the two subspecies using ANOVA ( $\mathrm{N}=31, \mathrm{~F}=12.212, \mathrm{p}=0.002)$. Homogeneity of variances was previously tested using Levene Statistic $(0.546<\mathrm{p}<0.959)$.

\section{DISCUSSION AND CONCLUSION}

Genetic differences among species and among subspecies (among populations of the same species inhabiting nonoverlapping geographic areas) can result in morphological differences. As vocalization parameters strongly depend on morphology of phonatory apparatus and on phonation mechanisms, we expect to be able to identify differences in acoustic parameters of calls emitted by different species [13, $31]$ but also by different subspecies [32, 33]. The present work showed that vocalizations are effective in highlighting differences not only between Eulemur species but also between Eulemur macaco subspecies, in accordance with genetic and morphological differences previously described [3, $8,9]$.

To perform this investigation we used the strategy of comparing the same type of vocalizations in the two different subspecies relying on data available from previous analyses [15].

The most important acoustic features for the differentiation of the two subspecies are those parameters affected by morphological characters of the vocal tract, formants. However, according to multivariate analysis the most informative parameters also include average Fundamental frequency, potentially reflective of different laryngeal structure, and duration.

These results indicate vocalizations may convey information that can inform receivers about the subspecies of the emitter. We also showed that this information could be successfully extracted using both larynx-related and vocal tractrelated parameters.

Moreover, vocal tract modelling based on acoustic parameters showed that blue-eyed black lemurs have a nasal tract significantly longer than the other subspecies, indicating a longer vocal tract presumably related to larger overall body size.

As previous studies on prosimians have shown, we do not expect the vocal repertoire of captive lemurs to be different from that exhibited by wild specimens in terms of call usage and vocal categories, though the calls can differ in frequency of emission $[10,16]$.

Continued study on the variability of acoustic features of vocalizations in the wild populations can offer insights on the correlation between changes in the acoustic structure, variation in genetics and external morphological traits of different populations or putative hybrids [32].

\section{ACKNOWLEDGEMENTS}

This research was supported in part by Parco Natura Viva - Garda Zoological Park, by the University of Torino, by the Ministero dell'Istruzione, dell'Università e della Ricerca (MIUR - Italy). We would like to thank Cesare Avesani Zaborra for his continuous support during the years. We greatly appreciated the help of Luca Pozzi and the support of the director and staff of the Parc Botanique et Zoologique de Tsimbazaza (Antananarivo, Madagascar) and of the Mulhouse Zoo (Mulhouse, France) in recording some subjects. We are also grateful to all other institutions that hosted the authors and allowed recordings.

\section{REFERENCES}

[1] Mittermeier RA, Tattersall I, Konstant WR, Meyers DM, Mast RB, Nash SD. Lemurs of Madagascar (Second Edition). Conservation International 2006.

[2] Schwitzer C, Schwitzer N, Randriatahina GH, Rabarivola C, Kaumanns W. "Programme Sahamalaza": New perspectives for the in situ and ex situ study and conservation of the blue-eyed black lemur (Eulemur macaco flavifrons) in a fragmented habitat. In: Schwitzer C, Brandt S, Ramilijaona O, Rakotomalala Razanahoera M, Ackermand D, Razakamanana T, Ganzhorn JU (Eds). Proceed- 
ings of the German-Malagasy Research Cooperation in Life and Earth Sciences 2006; 135-49.

[3] Rabarivola C. Etude génétique comparative de populations insulaires et "continentales" de Eulemur macaco. Utilisation simultanée des dermatoglyphes, de marqueurs sanguins et de l'ADN (RAPD) pour étudier la différenciation de E. macaco en deux sous-espèces: E.m. macaco et E.m. flavifrons (Thèse de doctorat d'Etat). Université d'Antananarivo 1998.

[4] Pastorini J. Molecular systematics of lemurs (dissertation). Universität Zürich 2000.

[5] Fausser JL, Rabarivola C, Meier B, Hahn T, Rumpler Y. Genetic comparison between different populations of Eulemur macaco flavifrons in northwest Madagascar using RAPD markers. Am J Primatol 2000; 51(4): 249-55.

[6] Koenders L, Rumpler Y, Ratsirarson J, Peyrieras A. Lemur macaco flavifrons (Gray, 1867): a rediscovered subspecies of Primates. Folia Primatol 1985; 44: 210-5.

[7] Meier B, Lonina A, Hahn T. Expeditionsbericht Sommer 1995 Schaffung eines neuen Nationalparks in Madagaskar. Zeitschrift des Kölner Zoo 1996; 39(2): 61-72.

[8] Meyers DM, Rabarivola C, Rumpler Y. Distribution and conservation of Sclater's lemur: Implications of a morphological cline. Prim Conserv 1989; 10: 77-81.

[9] Rabarivola C, Meyers D, Rumpler Y. Distribution and morphological characters of intermediate forms between the black lemur (Eulemur macaco macaco) and Sclater's lemur (Eulemur macaco flavifrons). Primates 1991; 32(2): 269-73.

[10] Macedonia JM, Stanger KF. Phylogeny of the Lemuridae revisited: evidence from communication signals. Folia Primatol 1994; 63: 143.

[11] Petter J-J, Charles-Dominique P. Vocal communication in prosimians. In: Doyle GA, Martin RD (Eds). The Study of Prosimian Behavior. Academic Press 1979; 247-305.

[12] Gosset D, Fornasieri I, Roeder JJ. Acoustic structure and contexts of emission of vocal signals by black lemurs. Evol Commun 2003; 4: 225-53.

[13] Gamba M, Giacoma C. Key issues in the study of primate acoustic signals. J Anthr Sci 2005; 83: 61-87.

[14] Gamba M, Pozzi L, Giacoma C. Lemurs uttering and its implications for phylogenetic reconstruction. In: Proceedings 5th ICVPB 2006; 123-26.

[15] Gamba M. Evoluzione della Comunicazione nei Lemuri del Madagascar (Tesi di Dottorato). Università degli Studi di Torino 2005.

[16] Fichtel C, van Schaik CP. Semantic differences in sifaka (Propithecus verreauxi) alarm calls: a reflection of genetic or cultural variants? Ethology 2006; 112: 839-49.
[17] Altmann J. Observational study of behavior: sampling methods. Behaviour 1974; 49: 227-67.

[18] Fant G. Acoustic theory of speech production. The Hague 1960.

[19] Titze IR. Principles of Voice Production. Prentice Hall 1994.

[20] Fitch WT. Vocal tract length and formant frequency dispersion correlate with body size in rhesus macaques. J Acoust Soc Am 1997; 102: 1213-22.

[21] Rendall D, Kollias S, Ney C, Lloyd P. Pitch (F0) and formant profiles of human vowels and vowel-like baboon grunts: The role of vocalizer body size and voice-acoustic allometry. J Acoust Soc Am 2005; 117 : 944-55.

[22] Gamba M, Giacoma C. Vocal tract modeling in a prosimian primate: the black and white ruffed lemur. Acta Acust United Ac 2006; 92: 749-55.

[23] Gamba M, Giacoma C. Quantitative acoustic analysis of the vocal repertoire of the crowned lemur. Ethol Ecol Evol 2007; 19(4): 32343.

[24] Gamba M, Medard J, Andriamialison H, Rakotoarisoa G, Giacoma C. Vocal tract modeling as a tool to investigate species-specific cues in vocalization. 2007, Proceedings 5th MAVEBA 2007; 2257.

[25] Boersma P, Weenink D. Praat: Doing phonetics by computer (Version. 4.3.20) 2006. Retrieved from: http://www.praat.org.

[26] Harris TR, Fitch WT, Goldstein LM, Fashing PJ. Black and white colobus monkey (Colobus guereza) roars as a source of both honest and exaggerated information about body mass. Ethology 2006; 112: 911-20.

[27] Charif RA, Mitchell S, Clark CW. Canary users' manual. Cornell Laboratory of Ornithology 1996.

[28] Plichta B. Akustyk for Praat. User manual. Michigan State University 2004.

[29] Fischer J, Hammerschmidt K, Cheney DL, Seyfarth RM. Acoustic features of female chacma baboon barks. Ethology 2001; 107: 3354.

[30] Reby D, McComb K. Anatomical constraints generate honesty: acoustic cues to age and weight in the roars of red deer stags. Anim Behav 2003; 65: 519-30.

[31] Zimmermann E, Vorobieva E, Wrogemann D, Hafen, T. Use of vocal fingerprinting for specific discrimination of gray (Microcebus murinus) and rufous mouse lemurs (Microcebus rufus). Int $\mathbf{J}$ Primatol 2000; 21: 837-52.

[32] Macedonia JM, Taylor LL. Subspecific divergence in a loud call of the ruffed lemur (Varecia variegata). Am J Primatol 1985; 9: 295304.

[33] Zimmermann E, Hafen TG. Colony specificity in a social call of mouse lemurs (Microcebus ssp). Am J Primatol 2001; 54: 129-41.

Received: March 20, 2008

Revised: April 29, 2008

Accepted: May 23, 2008

(C) Gamba and Giacoma; Licensee Bentham Open.

This is an open access article distributed under the terms of the Creative Commons Attribution License (http://creativecommons.org/licenses/by/2.5/), which permits unrestrictive use, distribution, and reproduction in any medium, provided the original work is properly cited. 